\title{
Gedruckte Quellen und Literatur
}

Abrassimow, Pjotr A.: 300 Meter vom Brandenburger Tor. Erinnerungen eines Botschafters, Berlin 1985.

Adibekov, G.M.: Kominform i poslevoennaja Evropa [Das Kominform und das Nachkriegseuropa], Moskva 1994.

Akinscha, Konstantin/Koslow, Grigori: Beutekunst. Auf Schatzsuche in russischen Geheimdepots, München 1995.

Aksen zur Auswärtigen Politik der Bundesrepublik Deutschland, Bd. I, Adenauer und die Hohen Kommissiare 1949-1951, hrsg. von Hans-Peter Schwarz, München 1989; Bd. 2, Adenaver und die Hohen Kommissare 1952, hrsg. von Hans-Peter Schwarz, München 1990.

Arbatow, Georgi: Das System. Ein Leben im Zentrum der Sowjetpolitik, Frankfurt/Main 1993.

Arlt, Kurt: Das Wirken der Sowjetischen Militäradministration in Deutschland (SMAD) im Spannungsfeld zwischen den Beschlüssen von Potsdam und den sicherheitspolitischen Interessen Moskaus 1945-1949, in: ThoB, Volksarmee, S. 91-139.

Die Auswärtige Politik der Bundesrepublik Deutschland, hrsg. vom Auswärtigen Amt, Köln 1972.

Badstübner, Rolf/Loth, Wilfried (Hrsg.): Wilhelm Pieck. Aufzeichnungen zur Deutschlandpolitik 1945-1953, Berlin 1994.

Badstïbner, Rolf: „Beratungen“ bei Stalin. Neue Dokumente, in: Utopie kreativ, Heft 7, März 1991, S. 99-116.

Badstübner, Rolf: Die sowjetische Deutschlandpolitik im Lichte neuer Quellen, in: Loth, Deutsche Frage, S. 102-135.

Badstübner, Rolf: Friedenssicherung und deutsche Frage. Vom Untergang des „Reiches“ bis zur deutschen Zweistaatlichkeit (1943 bis 1949), Berlin 1990.

Badstübner, Rolf: Zum Problem der historischen Alternativen im ersten Nachkriegsjahrzehnt. Neue Quellen zur Deutschlandpolitik der KPdSU und SED, in: BzG 33/1991, S. 579-592.

Bailey, George/Kondraschow, Sergej A/Murphy, David E.: Die unsichtbare Front. Der Krieg der Geheimdienste im geteilten Berlin, Berlin 1997.

Baring, Arnulf: Der 17. Juni 1953, Stuttgart 1983 (2).

Belezkij, Viktor N.: Die Politik der Sowjetunion in den deutschen Angelegenheiten in der Nachkriegszeit 1945-1976, Berlin 1977.

Bennewitz, Inge/Potratz, Rainer: Zwangsaussiedlungen an der innerdeutschen Grenze, Analysen und Dokumente, Berlin 1994.

Benser, Günter/Heitzer, Heinz: Die Gründung der DDR - Ergebnis einer erfolgreichen Volksbewegung, in: ZfG 26/1978, S. 209-213.

Bereschkow, Valentin M.: Ich war Stalins Dolmetscher. Hinter den Kulissen der politischen Weltbühne, München 1991.

Berija: Konec kar'ery, (Sammlung von Dokumenten, zusammengestellt und redigiert von V. F. Nekrasov), Moskva 1991.

Besier, Gerhard: Der SED-Staat und die Kirche. Der Weg in die Anpassung, München 1993.

Besymenski, Lew: 1953 - Berija will die DDR beseitigen, in: Die Zeit vom 15.10.1993, S. 81-83.

Bloch, Peter: Zwischen Hoffnung und Resignation. Als CDU-Politiker in Brandenburg 1945-1950, hrsg. von Siegfried Suckut, Köln 1986.

Bokow, Fjodor F.: Frühjahr des Sieges und der Befreiung. Berlin 1979.

Bonwetsch, Bernd: Deutschlandpolitische Alternativen der Sowjetunion, 1949 bis 1955, in: Deutsche Studien 22/1986, S. 320-340.

Bonwetsch, Bernd/Bordjugov, Gennadij: Die SED und die guten Erfahrungen der Sowjetunion: Pieck und Grotewohl informieren sich in Moskau, 25.3.-1.4.1948, in: Deutsche Studien 31/1994, S. 95-107.

Bonwetsch, Bernd/Bordjugov, Gennadij: Stalin und die SBZ. Ein Besuch der SED-Führung in Moskau vom 30. Januar bis 7. Februar 1947, in: VfZ 42/1994, S. 279-303.

Brandt, Heinz: Die sowjetische Deutschlandpolitik im Frühsommer 1953 aus der Sicht fortschrittlicher Kräfte in der SED, in: Osteuropa 15/1965, S. 369-377. 
Brzezinski, Zbigniew K.: Der Sowjetblock, Köln/Berlin (West) 1962.

Buchheim, Hans: Deutschlandpolitik 1949-1972. Der politisch-diplomatische ProzeB, Stuttgart 1984 (Schriftenreihe der V(Z, Bd. 49).

Buchheim, Christoph (Hrsg.), Wirtschaftliche Folgelasten des Krieges in der SBZ/DDR, Baden-Baden 1995.

Buddrus, Michael: ,... Im Allgemeinen ohne besondere Vorkommnisse“. Dokumente zur Situation des Strafvollzugs der DDR nach der Auflösung der sowjetischen Internierungslager 1949-1951, in: DA 29/1996, S. 10-33.

Buttlar, Walrab von: Ziele und Zielkonflikte der sowjetischen Deutschlandpolitik 1945-1947, Stuttgart 1980.

Childs, David: The GDR: Moscow's German Ally, London/Boston 1983.

Clay, Lucius D.: Decisions in Germany, Garden City 1950.

Cold War International History Project. Bulletin, hrsg. Woodrow Wilson International Center for Scholars, Washington, D.C., Fall 1994, Issue 4, p. 35, 48 (Dokumentenauszug, hrsg. v. M. M. Narinsky).

Creuzberger, Stefan: Die Sowjetische Militäradministration in Deutschland 1945-1949, Melle 1991.

Creuzberger, Stefan: Die sowjetische Besatzungsmacht und das politische System der SBZ, Weimar, Köln, Wien 1996.

Croan, Melvin: Dependent Totalitarism: The Political Process in East Germany, Ph. Diss., Harward 1960.

Cuev, Feliks: Sto sorok besed s Molotovym. Iz dnevnika F. Cueva [Hundertvierzig Gespräche mit Molotov. Aus dem Tagebuch von F. Cuev], Moskva 1991.

Die antifaschistisch-demokratische Umwälzung, der Kampf gegen die Spaltung Deutschlands und die Entstehung der DDR von 1945-1949, hrsg. von einem Autorenkollektiv (Rolf Badstübner, Evemarie BadstübnerPeters, Günter Benser, Peter Kirste, Siegfried Kuntsche, Wolfgang Mühlfriedel), Berlin 1989 (Deutsche Geschichte in zwölf Bänden, herausgegeben vom Zentralinstitut für Geschichte der Akademie der Wissenschaften der DDR, Band 9).

Die auswärtige Politik der Bundesrepublik Deutschland, hrsg. vom Auswärtigen Amt, Köln 1972.

Die Deutschlandfrage von der staatlichen Teilung bis zum Tode Stalins, Berlin 1994.

Die Entwicklung der freundschaftlichen Beziehungen zwischen der DDR und der UdSSR (Referate und Diskussionsbeiträge auf der XXII.Tagung der Kommission der Historiker der DDR und der UdSSR, 4./5. Juni 1974 in Berlin), Red. Harald Buttler, Berlin 1977.

Die ersten Jahre. Erinnerungen an den Beginn der revolutionären Umgestaltungen, eingeleitet und zusammengestellt von Ilse Schiel, 2. Auflage, Berlin 1985.

Die Sowjetunion und die Frage der Einheit Deutschlands und des Friedensvertrages mit Deutschland, Berlin 1952.

Diedrich, Torsten: Aufrüstungsvorbereitung und -finanzierung in der SBZ/DDR in den Jahren 1948 bis 1953 und deren Rückwirkung auf die Wirtschaft, in: Thoß, Volksarmee, S. 273-336.

Diedrich, Torsten: Der 17. Juni 1953 in der DDR. Bewaffnete Gewalt gegen das Volk, Berlin 1991.

Dijk, Ruud van: The German Question Dropped, Revisited and Frozen. The Kremlin, the East Germans and GDR in 1952-1953. Discussion paper prepared for the conference "Das Krisenjahr 1953 und der Kalte Krieg in Europa", Potsdam, November 10-12, 1996.

Doernberg, Stefan: Die Geburt eines neuen Deutschland 1945-1949. Die antifaschistisch-demokratische Umwälzung und die Entstehung der DDR, Berlin 1959.

Dokumente der Sozialistischen Einheitspartei Deutschlands, Bde. I-IV, Berlin 1951-1954.

Dokumente zur Außenpolitik der Regierung der DDR, hrsg. vom Deutschen Institut für Zeitgeschichte, Bd. 1, Berlin 1954.

Dokumente zur Deutschlandpolitik, II. Reihe, Bd. 2. Die Konstituierung der Bundesrepublik Deutschland and der Deutschen Demokratischen Republik, 7. September bis 31. Dezember 1949, hrsg. vom Bundesministerium des Innern, bearbeitet von Hanns Jürgen Küsters unter Mitarbeit von Daniel Hofmann, München 1996.

Dokumente zur Deutschlandpolitik der Sowjetunion, hrsg. vom Deutschen Institut für Zeitgeschichte, Bd. 1: Vom Potsdamer Abkommen am 2. August 1945 bis zur Erklärung über die Herstellung der Souveränität der Deutschen Demokratischen Republik am 25. März 1954, Berlin 1957.

Dokumente zur Entwicklung der Beziehungen der DDR und der UdSSR. 1949 bis zur Gegenwart, herausgegeben von der Akademie für Staats- und Rechtswissenschaften der DDR, Potsdam-Babelsberg, 1972. 
Dombrað̌va, T. V./Filitov, A. M.: Sovetskaja politika $\vee$ germanskom voprose (1953-1955) - tri osnovnych paradigma [Die sowjetische Politik in der deutschen Frage 1953-1955 - drei grundlegende Paradigmen], Manuskript von 1994.

Duhnke, Horst: Stalinismus in Deutschland. Die Geschichte der Sowjetischen Besatzungszone, Köln 1955.

Dunmore, Thimothy: Soviet Politics 1945-53, New York 1984.

Dymschitz, Alexander (Lwowitsch): Ein unvergeBlicher Frühling. Erinnerungen eines sowjetischen Kulturoffiziers, Berlin 1970.

Eisert, Wolfgang: Die Waldheimer Prozesse. Der stalinistische Terror 1950. Ein dunkles Kapitel der DDR-Justiz, München 1993.

Erler, Peter/Otto, Wilfriede/Prieß, Lutz: Sowjetische Internierungslager in der SBZ/DDR 1945 bis 1950, in: BzG 32/1990, S. 723-734.

Ersil, Wilhelm: Adenauers Verhandlungen mit den Hohen Kommissaren und die Politik gegenüber der DDR, in: Scherstjanoi, Provisorium, S. 163-169.

Falin, Valentin: Politische Erinnerungen, München 1993

Fejto, Francois: Die Geschichte der Volksdemokratien, Buch 1 - Ära Stalin 1945-1953, Frankfurt/Main 1988[2].

Filipovych, D.N.: Sovetskaja Voennaja Administracija v Germanii: Voenno-politiceskij aspekt dejatel'nosti (1945-1949 gg.) [Die Sowjetische Militäradministration in Deutschland: der militärpolitische Aspekt ihrer Tätigkeit (1945-1949)], Moskva 1995.

Filitov, Alexej M.: The Soviet Policy and Early Years of Two German States, 1949-1961, Referat auf der Konferenz zur sowjetischen Deutschlandpolitik, Essen Juni 1994, unveröffentlichtes Skript).

Filitov, Alexej M.: V komissijach Narkomindela [In den Kommissionen des Narkomindel (Volkskommissariats für Äußere Angelegenheiten)], in: Vtoraja mirovaja vojna. Aktual'nye problemy [Der Zweite Weltkrieg. Aktuelle Probleme], Moskva 1995, S. 54-71.

Filitov, Alexej M.: Germanskij vopros: ot raskola k ob"'edineniju. Novoe proctenie [Die deutsche Frage: von der Teilung bis zur Vereinigung. Eine neue Lesart], Moskva 1993.

Filitov, Alexej M.: Perelomnyj 1953-yj: Dynamika peremen v mirovoj i sovetskoj vnešnej politike [Das Wendejahr 1953: Die Dynamik der Veränderungen in der internationalen Politik und der sowjetischen Außenpolitik], Vortrag auf der Konferenz „Das Krisenjahr 1953 und der Kalte Krieg in Europa“, Potsdam, 10.-12. November 1996.

Finley, David D.: Soviet Foreign Policy Decision Making, Stanford, Ph. Diss. 1966.

Fisch, Jörg: Reparationen nach dem Zweiten Weltkrieg, München 1992.

Fischer, Alexander/Lipinsky, Jan: Die sowjetischen Speziallager Buchenwald und Fünfeichen - Erkenntnisse aus sowjetischen Archiven, in: Deutsche Studien 31/1994, S. 38-56.

Fischer, Alexander: Die Sowjetunion und die „deutsche Frage“ 1945-1949, in: Die Deutschlandfrage und die Anfänge des Ost-West-Konflikts 1945-1949, Berlin 1984, S. 41-57.

Flocken, Jan von/Klonovsky, Michael: Stalins Lager in Deutschland 1945-1950. Dokumentation, Zeugenberichte, Berlin, Frankfurt/Main 1991.

Flocken, Jan von/Scholz, Michael F.: Ernst Wollweber. Saboteur Minister Unperson, Berlin 1994.

Foitzik, Jan: Berichte des Hohen Kommissars der UdSSR in Deutschland aus den Jahren 1953/54. Dokumente aus dem Archiv für AuBenpolitik der Russischen Föderation, in: Materialien der Enquete-Kommission "Aufarbeitung von Geschichte und Folgen der SED-Diktatur in Deutschland" (12. Wahlperiode des deutschen Bundestages), Bd. 11.2, S. 1350-1541.

Foitzik, Jan: Sowjetische Militäradministration in Deutschland (SMAD), in: SBZ-Handbuch: staatliche Verwaltungen, Parteien, gesellschaftliche Organisationen und ihre Führungskräfte in der Sowjetischen Besatzungszone Deutschlands 1945-1949, hrsg. von Martin Broszat u. Hermann Weber, München 1990, S. 7-69.

Foitzik, Jan: Befehls- und Kommunikationsstruktur der Sowjetischen Militäradministration in Deutschland (SMAD), in: Schönhoven, Klaus/Staritz, Dietrich (Hrsg.): Sozialismus und Kommunismus im Wandel: Hermann Weber zum 65. Geburtstag, Köln 1993, S. 324-351.

Foitzik, Jan: Zur Situation in Moskauer Archiven, in: Jahrbuch für historische Kommunismusforschung 1993, Berlin 1993, S. 299-308.

Foitzik, Jan: Sowjetische Hegemonie und Kommunismus in Ostmitteleuropa nach dem Zweiten Weltkrieg, in: Aus Politik und Zeitgeschichte, Beilage zur Wochenzeitschrift Das Parlament, B 37-38/96, S. 29-37. 
Foschepoth, Josef (Hrsg.): Adenauer und die Deutsche Frage, Göttingen, Zürich 1988

Fragen des Aufbaus eines einheitlichen, demokratischen, friedliebenden deutschen Staates (Übersetzung aus dem Russischen), Berlin 1952.

Freundschaft DDR - UdSSR. Dokumente und Materialien, Berlin 1965.

Freundschaft für immer. Deutsche Genossen berichten über die Tradition der deutsch-sowjetischen Freundschaft, Berlin 1960.

Freundschaft - Werden und Wachsen (1945-1949). Ausgewählte Dokumente und Materialien zur Entwicklung des Freundschafts- und Bruderbundes zwischen der Sowjetunion und der DDR, eingeleitet und ausgewählt von Friedrich Beck. Kurt Libera, Hans-Joachim Schreckenbach und Wemer Völk, Potsdam 1975.

Fricke, Karl Wilhelm/Marquardt, Bernhard: DDR-Saatssicherheit. Das Phänomen des Verrats - Die Zusammenarbeit zwischen MfS und KGB, Bochum 1995.

Fricke, Karl Wilhelm: Politik und Justiz in der DDR. Zur Geschichte der politischen Verfolgung 1945-1968, Köln 1979.

Fritsch-Bournazel, Renata: Die Sowjetunion und die deutsche Teilung. Die sowjetische Deutschlandpolitik von 1945-1979, Opladen 1979.

Gall, Wladimir: Mein Weg nach Halle, Berlin 1988.

Germanskaja Demokraticeskaja Respublika (nekotorye dokumenty i materialy) [Die Deutsche Demokratische Republik (einige Dokumente und Materialien)], hrsg. vom Otdel informacii Sovetskoj Kontrol'noj Komissii v Germanii [Abteilung für Information der Sowjetischen Kontrollkommission in Deutschland], Berlin 1952.

Geyer, Dietrich: Von der Kriegskoalition zum Kalten Krieg, I: 1917-1955, in: Osteuropa-Handbuch, Sowjetunion, Außenpolitik 1917-1955, Köln und Wien 1972, S. 341-383.

Geyer, Dietrich: Deutschland als Problem der sowjetischen Europapolitik am Ende des Zweiten Weltkrieges, in: Foschepoth, Josef (Hrsg.), Kalter Krieg und deutsche Frage. Deutschland im Widerstreit der Mächte 1945-1952, Göttingen/Zürich 1985, S. 50-65.

Glaser, Günther: „Reorganisation der Polizei“ oder getarnte Bewaffnung der SBZ im Kalten Krieg? Dokumente und Materialien zur sicherheits- und militärpolitischen Weichenstellung in Ostdeutschland 1948/49. Frankfurt/Main 1995.

Gniffke, Erich W.: Jahre mit Ulbricht, Köln 1966.

Graml, Hermann: Die Legende von der verpaßten Gelegenheit, in: VfZ 29/1981, S. 307-341.

Gromyko, Andrej: Memories, London 1989.

Haffner, Friedrich: Das System der Leitung und Planung der Volkswirtschaft in der DDR und in der Sowjetunion, in: Mampel, Siegfried/Thalheim, Karl C. (Hrsg.): Die DDR. Partner oder Satellit der Sowjetunion (Jahrbuch der Gesellschaft für Deutschlandforschung 1979), München 1980, S. 73-114.

Hagen, Manfred: DDR - Juni '53: die erste Volkserhebung im Stalinismus, Stuttgart 1992.

Hahn, Werner G.: Postwar Soviet Politics. The Fall of Zhdanov and the Defeat of Moderation, 1946-1953, Ithaca, London 1982.

Hajna, Karl-Heinz: Länder - Bezirke - Länder. Zur Territorialstruktur im Osten Deutschlands 1945-1990, Frankfurt/Main u.a. 1995.

Hänisch, Werner: Außenpolitik und intemationale Beziehungen der DDR, Bd. 1, 1949-1955, Berlin 1972.

Harrison, Hope M.: The Bargaining Power of Weaker Allies in Bipolarity and Crisis: The Dynamics of SovietEast German Relations, 1953-1961, Dissertation, Columbia University 1993.

Hegemann, Margot: Zum Prozeß der Verankerung der DDR in der sozialistischen Staatengemeinschaft, in: Die DDR in der Übergangsperiode. Studien zur Vorgeschichte und Geschichte der DDR 1945 bis 1961 , hrsg. von Rolf Badstïbner und Heinz Heitzer, 2. Auf., Berlin 1979, S. 85-115.

Heider, Magdalena: Politik - Kultur - Kulturbund. Zur Gründungs- und Frühgeschichte des Kulturbundes zur demokratischen Erneuerung Deutschlands 1945-1954 in der SBZ/DDR, Köln 1993.

Heitzer, Heinz: Die Bildung der Provisorischen Regienung, in: Scherstjanoi, Provisorium, S. 313-321.

Henke, Josef/Oldenhage, Klaus: Office of Military Government for Germany (U.S.), in: Weisz, Christoph (Hrsg): OMGUS-Handbuch. Die amerikanische Militärregierung in Deutschland 1945-1949, München 1994, S. $1-142$.

Herbst, Ludolf: Abhängigkeit oder Interdependenz? (Kommentar), in: Kocka, Jürgen/Sabrow, Martin (Hrsg.): Die DDR als Geschichte. Fragen - Hypothesen - Perspektiven, Berlin 1994, S. 186-190. 
Hershberg, James G.: Soviet Archives: The Opening Door, in Bulletin of the Cold War International History Project, Issue 1, Woodrow Wilson International Center of Scholars, Washington D.C., Spring 1992, S. 1, 12-15, 23-27.

Hillgruber, Andreas: Alliierte Pläne für eine „Neutralisierung“ Deutschlands 1945-1955, Opladen 1987.

Hillgruber, Andreas: Europa in der Weltpolitik der Nachkriegszeit 1945-1963, 4. Aufl., München 1993.

History of the Allied High Comission for Germany. Its Establishment, Structure and Procederes, Frankfurt/ Main 1951.

Hodos, Georg Hermann: Schauprozesse. Stalinistische Säuberungen in Osteuropa 1948-54, Frankfurt/Main, New York 1988.

Holzwarth, Klaus: Die Anfänge der zentralen Wirtschaftsplanung in der SBZ, in: Buchheim, Christoph (Hrsg.): Wirtschaftliche Folgelasten des Krieges in der SBZ/DDR, Baden-Baden 1995, S. 247-269.

Inventar der Befehle des Obersten Chefs der Sowjetischen Militäradministration in Deutschland (SMAD) 1945-1949. Offene Serie, zusammengestellt und bearbeitet von Jan Foitzik, München u.a. 1995.

Izvestija CK KPSS, 1990, Hefte 1 und 2.

Jäckel, Eberhard (Hrsg.): Die deutsche Frage 1952-1956, Notenwechsel und Konferenzdokumente der vier Mächte, Frankfurt/Main, Berlin 1957.

Jarausch, Konrad/Siegrist, Hannes (Hrsg.): Amerikanisierung und Sowjetisierung in Deutschland 1945-1970, Frankfurt/Main u.a. 1997.

Jones, Christopher D.: Soviet Influence in Eastem Europe: Political Autonomy and Warsaw Pact, New York 1981.

Kaiser, Monika (Beitrag auf einer Podiumsdiskussion 1993): Entstalinisierungskrise und Parteiführung der SED, in: Kocka, Jürgen/Sabrow, Martin (Hrsg.): Die DDR als Geschichte, Berlin 1994, S. 50-52.

Kaiser, Monika: Sowjetischer Einfluß auf die ostdeutsche Politik und Verwaltung, in: Jarausch/Siegrist, Amerikanisierung, S. 111-133.

Kampfgefährten - Weggenossen. Erinnerungen deutscher und sowjetischer Genossen an die ersten Jahre der antifaschistisch-demokratischen Umwälzung in Dresden, hrsg. von Helfried Wehner in Zusammenarbeit mit Anatoli Waks, Berlin 1975.

Kampfgemeinschaft SED-KPdSU. Grundlagen, Traditionen, Wirkungen. Referate und Diskussionsbeiträge. XXIV. Tagung der Kommission der Historiker der DDR und der UdSSR 8./9. Dezember 1975 in Moskau, Berlin 1978.

Kanzig, Helga: Die Anwendung der Erfahrungen und die Zusammenarbeit mit der UdSSR beim Aufbau des Sozialismus in der DDR, in: Jahrbuch für Wirtschaftsgeschichte IV/1977, S. 9-25.

Karjagin, V.: Berlin ot żeleznogo zanavesa do betonnoj steny [Berlin vom Eisernen Vorhang zur Mauer aus Beton], in: (1) Meždunarodnaja żizn, 1995, Heft 4, S. 94-106, (2) Meždunarodnaja żizn, 1995, Heft 5 , S. 85-98, (3) Meždunarodnaja žizn, 1995, Heft 6, S. 91-104.

Karlsch, Rainer: Allein bezahlt? Die Reparationsleistungen der SBZJDR 1945-53, Berlin 1993.

Karlsch, Rainer: Eine wichtige neue Quelle für die Wirtschaftsgeschichte der SBZ/DDR von 1945 bis 1955. Der Bestand der Garantie- und Kreditbank AG, in: Archivmitteilungen 42/1993, S. $107 \mathrm{f}$.

Kießling, Wolfgang: Der Fall Baender. Ein Politkrimi aus den 50er Jahren, Berlin 1991.

Kießling, Wolfgang: Partner im Narrenparadies. Der Freundeskreis um Noel Field und Paul Merker, Berlin 1994.

Kirchliches Jahrbuch 1951.

KleBmann, Christoph: Die doppelte Staatsgründung. Deutsche Geschichte 1945-1955, 5. überarb. und erw. Auflage, Göttingen 1991.

Klimow, Gregory: Berliner Kreml, Köln 1952.

Knight, Amy: Beria. Stalin's First Lieutenant, Princeton 1993.

Knoll, Viktor/Kölm Lothar: Der Fall Berija. Protokoll einer Abrechnung. Das Plenum des ZK der KPdSU, Juli 1953, Stenographischer Bericht (Übersetzung aus Izvestija CK KPSS, 1990, Nr.1, 2).

Knyscheskij, Pawel Nikolajewitsch: Moskaus Beute. Wie Vermögen, Kulturgüter und Intelligenz nach 1945 aus Deutschland geraubt wurde, München 1995.

Kollner, Lutz/Maier Klaus A. u.a.: Anfänge westdeutscher Sicherheitspolitik 1945-1956, (hrsg. vom Militärgeschichtlichen Forschungsamt), Band 2: Die EVG-Phase, München 1990. 
Kokurin, A. I./Požarov, A. I. (Hrsg.): „Novyj kurs“ L. P. Berii 1953 g. [Der „neue Kurs“ Berijas im Jahr 1953], in: Istoriceskij archiv 4 [1996], Nr. 4, S. 132-164.

Koval', K. I.: Robota v Germanii po zadaniju GKO [Arbeit in Deutschland im Auftrag des Staatlichen Komitees für Verteidigung], in: Novaja i novejšaja istorija, 1995, Heft 2, S. 101-114.

Koval', K. I.: Zapiski upolnomocennogo GKO na territorii Germanii [Notizen eines Beauftragten des Staatlichen Komitees für Verteidigung auf dem Territorium Deutschlands], in: Novaja i novejšaja istorija, 1994, Heft 3, S. 124-147.

Koval, K. 1.: Mein Treffen mit General Draper im Juli 1947, in: BzG 37/1995, Heft 2, S. 41-61.

Kreikamp, Hanns-Dieter (Hrsg.): Quellen zur staatlichen Neuordnung Deutschlands 1945-1949, Darmstadt 1994.

Krisch, Henry: German Politics under Soviet Occupation, New York 1974.

Krushchev Remembers. The Glasnost Tapes, Boston 1990.

Küsters, Hanns Jürgen: Einleitung zu: Dokumente zur Deutschlandpolitik (1996).

Künzel, Werner: Das Ministerium der Justiz im Mechanismus der Justizsteuerung 1949 bis 1976, in: Rottleuthner, Hubert: Steuerung der Justiz in der DDR: Einflußnahme der Politik auf Richter, Staatsanwalte und Rechtsanwälte, Köln 1994, S. 167-252.

Künzel, Werner: Die DDR-Gründung im Spiegel ostdeutscher Landtagsdebatten, in: Scherstjanoi, Provisorium, S. 327-334.

Kwizinskij, Julij A.: Vor dem Sturm, Erinnerungen eines Diplomaten, Berlin 1993.

Laufer, Jochen: Auf dem Wege zur staatlichen Verselbständigung der SBZ. Neue Quellen zur Münchener Konferenz der Ministerpräsidenten 1947, in: Kocka, Jürgen (Hrsg.): Historische DDR-Forschung. Aufsätze und Studien, Berlin 1993, S. 27-55.

Laufer, Jochen: Die DDR - ein gewollter staatlicher Neuanfang? (Thesen), in: Kocka, Jürgen/Sabrow, Martin (Hrsg.): Die DDR als Geschichte. Fragen - Hypothesen - Perspektiven, Berlin 1994, S. 177-180.

Laufer, Jochen: Die SED und die Wahlen (1949-1950). Untersuchung zu den politischen Entscheidungsprozessen, in: Scherstjanoi, Provisorium, S. 101-124.

Laufer, Jochen: Konfrontation oder Kooperation? Zur sowjetischen Politik in Deutschland und im Alliierten Kontrollrat 1945-1948, in: Fischer, Alexander (Hrsg.): Studien zur Geschichte der SBZ/DDR, Berlin 1993, S. 57-80.

Lederer, Ivo (Hrsg.): Russian Foreign Policy, New Haven, London 1962.

Lemke, Michael: „Doppelte Alleinvertretung“. Die nationalen Wiedervereinigungskonzepte der beiden deutschen Regierungen und die Grundzüge ihrer politischen Realisierung in der DDR (1949-1952/53), in: ZfG 40/1992, S. 531-543.

Lemke, Michael: Die Deutschlandpolitik der DDR zwischen Moskauer Oktroi und Bonner Sogwirkung, in: Kocka, Jürgen/Sabrow, Martin (Hrsg.): Die DDR als Geschichte. Fragen - Hypothesen - Perspektiven, Berlin 1994, S. 181-185.

Lemke, Michael: Die DDR und die deutsche Frage 1949-1955, in: Loth, Deutsche Frage, S. 136-171.

Lipinsky, Jan: Sowjetische Speziallager in Deutschland 1945-1950 - ein Beispiel für alliierte Internierungspraxis oder für sowjetisches GULAG-System, in: Kaff, Brigitte (Hrsg.), „Gefährliche politische Gegner". Widerstand und Verfolgung in der sowjetischen Zone/DDR, Düsseldorf 1995, S. 27-43.

Lippmann, Heinz: Honecker. Porträt eines Nachfolgers, Köln 1971.

Lokatis, Siegfried: Dietz. Probleme der Ideologiewirtschaft im zentralen Parteiverlag der SED, in: Jansen, Christian/Niethammer, Lutz/Weisbrodt, Bemd: Von der Aufgabe der Freiheit. Politische Verantwortung und bürgerliche Gesellschaft im 19. und 20. Jahrhundert. Festschrift für Hans Mommsen zum 5 . November 1995, Berlin 1995, S. 533-548.

Lorenz, Thomas: Die Deutsche Zentralverwaltung der Justiz (DJV) und die SMAD in der sowjetischen Besatzungszone 1945 bis 1949, in: Rottleuthner, Hubert (Hrsg.): Steuerung der Justiz in der DDR. Einflußnahme der Politik auf Richter, Staatsanwälte und Rechtsanwälte, Köln 1994, S. 135-165.

Loth, Wilfried (Hrsg.): Die deutsche Frage in der Nachkriegszeit, Berlin 1994.

Loth, Wilfried: Die Teilung der Welt. Geschichte des Kalten Krieges 1941-1955, München 1980.

Loth, Wilfried: Stalins ungeliebtes Kind. Warum Moskau die DDR nicht wollte, Berlin 1994. 
Mackintosh, John M.: Strategy and Tactics of Soviet Foreign Policy, New York 1962.

Mai, Gunther: Der Alliierte Kontrollrat in Deutschland, München 1995.

Malycha, Andreas: Partei von Stalins Gnaden? Die Entwicklung der SED zur Partei neuen Typs in den Jahren 1946 bis 1950 . Berlin 1996.

Mampel, Siegfried/Thalheim, Karl C. (Hrsg.): Die DDR. Partner oder Satellit der Sowjetunion, München 1980.

Mampel, Siegfried: Die Entwicklung der Verfassungsordnung in der Sowjetzone Deutschlands von 19451963, in: Jahrbuch des öffentlichen Rechts der Gegenwart. N.F. Bd. 13, Tübingen 1964, S. 455-579.

Marcou, Lilly: Le Kominform. Le communisme de guerre froide, Paris 1977.

Marquardt, Bernhard: Die Zusammenarbeit zwischen MfS und KGB, in: Fricke, Karl Wilhelm/Marquardt, Bemhard: DDR-Staatssicherheit. Das Phänomen des Verrats. Die Zusammenarbeit zwischen MFS und KGB, Bochum 1995, S. 50-169.

Mastny, Vojtech: Missed Opportunities After Stalin's Death?, Paper prepared for conference on „Das Krisenjahr 1953 und der Kalte Krieg in Europa", Potsdam, 10-12 November 1996.

Mastny, Vojtech (Hrsg.): East European Dissent, vol. 1: 1953-1964, New York 1972.

Mastny, Vojtech: Moskaus Weg zum Kalten Krieg, München/Wien 1981.

Matke, Fritz: Starthilfe für neue Literatur. Altes und neues vorn SWA-Verlag, Börsenblatt für den Deutschen Buchhandel, 1989, Hefte 40-42.

Meissner, Boris: Sowjetunion. Der Auswärtige Dienst der UdSSR, in: Osteuropa 5/1955, S. 42-44.

Meissner, Boris: Rußland, die Westmächte und Deutschland. Die sowjetische Deutschlandpolitik 1943-1953, Hamburg 1953.

Mittenzwei, Werner: Das Leben des Bertolt Brecht oder Der Umgang mit den Welträtseln. Zweiter Band, Berlin 1988.

Müller-Enbergs, Helmut: Der Fall Rudolf Hermstadt. Tauwetterpolitik vor dem 17. Juni 1953, Berlin 1991.

Münch, Ingo von (Hrsg.): Dokumente des geteilten Deutschlands, Bd. 1, Stuttgart 1968.

Naimark, Norman M.: The Russians in Germany. A History of the Soviet Zone of Occupation, 1945-1949, Cambridge u.a. 1995.

Naimark, Norman M.: Die Russen in Deutschland. Die sowjetische Besatzungszone 1945 bis 1949, Berlin 1997.

Naimark, Norman M.: Die sowjetische Militäradministration in Deutschland und die Frage des Stalinismus. Veränderte Sichtweisen auf der Grundlage neuer Quellen aus russischen Archiven, in: ZfG 43/1995, S. 293-307.

Neef, Helmut: Entscheidende Tage im Oktober 1949. Die Gründung der Deutschen Demokratischen Republik, Berlin 1984.

Nettl, J.P.: The Eastern Zone and Soviet Policy in Germany 1945-50, London/New York 1951.

New Russian Documents on the Korean War, introduction and translation by Kathryn Weatherby, in: Cold War International History Project, Bulletin Issues 6-7. Woodrow Wilson International Center for Scholars, Washington D.C., Winter 1995/1996, S. 30-40.

Niedbalski, Bernd: Die kaderpolitischen und staatsorganisatorischen Voraussetzungen für die Bildung der DDR-Regierung 1949, in: Scherstjanoi, Provisorium, S. 305-312.

Niekisch, Ernst: Erinnerungen eines deutschen Revolutionärs. Zweiter Band: Gegen den Strom, 1945-1967, Köln 1974 .

Novik, F.: Sovetskaja politika v otnošenii GDR do i posle 17 ijunja 1953 goda [Die sowjetische Politik im Verhältnis zur DDR vor und nach dem 17. Juni 1953], Vortrag auf der Konferenz „Das Krisenjahr 1953 und der Kalte Krieg in Europa", Potsdam, 10.-12. November 1996.

Osten, Walter: Die Deutschlandpolitik der Sowjetunion in den Jahren 1952/53, in: Osteuropa 14/1964, Heft 1, S. $1-13$.

Otto, Wilfriede: Die „Waldheimer Prozesse“ 1950. Historische, politische und juristische Aspekte im Spannungsfeld zwischen Antifaschismus und Stalinismus, in: hefte zur ddr-geschichte, 12, Berlin 1993, S. 5-27.

Otto, Wilfriede: Dokumente zur Auseinandersetzung in der 1953, in: BzG 32/1990, S. 655-672.

Otto, Wilfriede: Sowjetische Deutschlandnote 1952. Stalin und die DDR. Bisher unveröffentlichte handschriftliche Notizen Wilhelm Piecks, in: BzG 33/1991, S. 374-389. 
Otto, Wilfriede: Sowjetische Deutschlandpolitik 1952/53. Forschungs- und Wahrheitsprobleme, in: DA 26/1993, S. 948-954.

Otto, Wilfriede: Deutscher Handlungsspielraum und sowjetischer EinfluB. Zur Rolle der Sowjetischen Kontrollkommission, in: Scherstjanoi, Provisorium, S. 138-144.

Overesch, Manfred: Die Deutschen und die Deutsche Frage 1945 - 1955, Hannover 1985.

Podewin, Norbert: Walter Ulbricht. Eine neue Biographie, Berlin 1995.

Pontifex nicht Partisan. Kirche und Staat in der DDR von 1949 bis 1958 . Dokumente aus der Arbeit des Bevollmächtigten des Rates der EKD bei der Regierung der DDR, Propst Dr. Heinrich Grüber, Stuttgart 1974.

Programmatische Dokumente der Nationalen Front des demokratischen Deutschland, hrsg. u. eingel. von Helmut Neef, Berlin 1967.

Protokoll der Verhandlungen der II. Parteikonferenz der Sozialistischen Einheitspartei Deutschlands, 9.-12. Juli 1952, Berlin 1952.

Protokoll des III. Parteitages der Sozialistischen Einheitspartei Deutschlands, 20. bis 24. Juli 1950 in der Werner-Seelenbinder-Halle zu Berlin, (2 Bde.), Berlin 1951.

Ra'anan, Gavriel D.: Intemational Policy Formation in the USSR. Factional „Debates“ during the Zhdanovshina, Hamden, Conn. 1983.

Rakitin, P.M./Sjas'ko, V.B./Ivanovskij, E.F.(Leiter des Redaktionskollegiums): Na boevom postu. Kniga o voinach Gruppy sovetskich vojsk v Germanii [Auf Gefechtsposten. Ein Buch über die Kämpfer der Gruppe der Sowjetischen Streitkräfte in Deutschland], Moskva 1975.

Resin, Leonid: Sobytija 17 ijunija 1953 goda v GDR - v zerkale Moskovskich archivov [Die Ereignisse des 17. Juni 1953 im Spiegel Moskauer Archive], Vortrag auf der Konferenz „Das Krisenjahr 1953 und der Kalte Krieg in Europa“, Potsdam, 10.-12. November 1996.

Richter, James: Reexamining Soviet policy towards Germany During the Beria Interregnum (Working Paper Nr. 3, Woodrow Wilson Center), 1992.

Richter, James: Reexamining Soviet Policy towards Germany during the Beria Interregnum, Paper prepared for delivery at the Conference on "New Evidence in the History of the Cold War", Moscow, January 12-15, 1993, Lewiston 1993.

Richter, Michael: Die Rolle der CDU der SBZ in der sowjetischen Deutschlandpolitik in den Jahren 19491952, in: Deutschlandfrage, S. 155-181.

Richter, Michael: Die Ost-CDU 1948-1952. Zwischen Widerstand und Gleichschaltung, Sankt Augustin 1991(2).

Ritscher, Bodo: Die NKWD/MWD-,,Speziallager" in Deutschland. Anmerkungen zu einem Forschungsgegenstand, in: Danyel, Jürgen (Hrsg.): Die geteilte Vergangenheit. Zum Umgang mit Nationalsozialismus und Widerstand in beiden deutschen Staaten, Berlin 1995, S. 163-179.

Ritscher, Bodo: Zur Herausbildung und Organisation des Systems von Speziallagern des NKFD in der sowjetischen Besatzungszone im Jahre 1945, in: DA 26/1993, S. 723-735.

Rodenbach, Hermann-Josef: Die verstärkte Sowjetisierungspolitik Moskaus und die Entwicklung der DDR, in: Deutschlandfrage, S. 133-154.

Roesler, Jörg: Die Herausbildung der sozialistischen Planwirtschaft in der DDR. Aufgaben, Methoden und Ergebnisse der Wirtschaftsplanung in der zentralgeleiteten volkseigenen Industrie während der Übergangsperiode vom Kapitalismus zum Sozialismus, Berlin 1978.

Rošcin, A. A.: Poslevoennoe uregulirovanie v Evrope [Die Nachkriegsregelung in Europa], Moskva 1984.

Rossija. XX vek. Dokumenty. Lubjanka 1917-1960. Spravočnik, Red. R. G. Pichoja, Moskva 1997.

Roßmann, Gerhard: Die Kampfgemeinschaft der SED und der KPdSU - Kraftquell für das Gedeihen der sozialistischen DDR, in: Die Entwicklung der freundschaftlichen Beziehungen, S. 53-65.

Rubinstein, Alvin Z: Stalins Postwar Foreign Policy in Perspective: A Review Article, in: Joumal of Conflict Resolutions, VIII, 2 (Juni) S. 186-194.

Rudolph, Vladimir: The Administrative Organisation of Soviet Control 1945-1948, in: Slusser, Soviet Economic Policy, S. 18-86.

Rupieper, Hermann-Josef: Der besetzte Verbündete. Die amerikanische Deutschlandpolitik 1949-1953, Opladen 1991.

Rupieper, Hermann-Josef: Zu den sowjetischen Deutschlandnoten 1952. Das Gespräch Stalin - Nenni, in: VfZ $33 / 1985$, S. 547-557. 
Russkij archiv. Velikaja Otecestvennaja: Bitva za Berlin (Krasnaja Armija v poveržennoj Germanii) [Russisches Archiv. Der Große Vaterländische Krieg: Der Kampf um Berlin (Die Rote Armee im besiegten Deutschland], Band 15 (4-5), Moskva 1995.

SBZ-Handbuch. Staatliche Verwaltungen, Parteien, geselischaftliche Organisationen und ihre Führungskräfte in der Sowjetischen Besatzungszone Deutschlands 1945-1949, hrsg. von Martin Broszat und Hermann Weber, München 1990.

Schenk, Fritz: Das rote Wirtschaftswunder. Die zentrale Planwirtschaft als Machtmittel der SED-Politik, Stuttgart 1969.

Schenk, Fritz: Im Vorzimmer der Diktatur. 12 Jahre Pankow, Köln, Berlin, 1962.

Schenk, Fritz: Magie der Planwirtschaft, Köln 1960.

Scherstjanoi, Elke/Laufer, Jochen: Erste Schritte zur Öffnung des Bestandes der Sowjetischen Militäradministration in Deutschland (SMAD), in: Archivmitteilungen 40/1990, S. 172-175.

Scherstjanoi, Elke/Stappenbeck, Christian: „Dibelius war in Karlshorst..., wollte Mittelsmann sein zwischen SKK und Adenauer..." Ein geheimes Gespräch zwischen Bischof Dibelius, Armeegeneral Tschujkow und Politberater Semjonow im November 1951, in: DA 28/1995, S. 1031-1047.

Scherstjanoi, Elke: „Provisorium für längstens ein Jahr." Die Gründung der DDR (Kolloquium 1991), Berlin 1993.

Scherstjanoi, Elke: „Wollen wir den Sozialismus?“ Dokumente aus der Sitzung des Politbüros des ZK der SED am 6. Juni 1953, in: BzG 33/1991, S. 658-680.

Scherstjanoi, Elke: Die DDR im Frühjahr 1952. Sozialismuslosung und Kollektivierungsbeschluß in sowjetischer Perspektive, in: DA 27/1994, S. 354-363.

Scherstjanoi, Elke: Friedenshektarerträge auf Vorkriegsanbauflächen. Zur Bewältigung von Kriegslasten in der ostdeutschen Landwirtschaft, in: Buchheim, Christoph (Hrsg.): Folgelasten, S. 323-340.

Scherstjanoi, Elke: Paul Baender und die „zuständigen“ Offiziere der sowjetischen Kontrollkommission, in: BzG 35/1993, Heft 4, S. 80-98.

Scherstjanoi, Elke: Zur aktuelien Debatte um die Stalin-Note 1952, in: DA 27/1994, S. 181-185.

Schirdewan, Karl: Aufstand gegen Ulbricht. Im Kampf um politische Kurskorrektur, gegen stalinistische, dogmatische Politik, 2. Auflage, Berlin 1994.

Schöneburg, Karl-Heinz u.a.: Vom Werden unseres Staates. Eine Chronik. Bd. 1 (1945-1949), Berlin 1966, Bd. 2 (1949-1955), Berlin 1968.

Schollwer, Wolfgang: Potsdamer Tagebuch 1948-1950, Liberale Politik unter sowjetischer Besatzung, hrsg. von Monika Faßbaender, München 1988.

Schwarz, Hans Peter (Hrsg.): Akten zur Auswärtigen Politik der Bundesregierung, Bd. I: Adenauer und die Hohen Kommissare 1949-1951, München 1989.

Schwarz, Hans Peter: Die Ära Adenauer. Die Gründerjahre der Republik 1949-1957, Stuttgart, Wiesbaden 1981.

Selbmann, Fritz: Die UdSSR unterstützte den Wiederaufbau des Wirtschaftslebens, in: Zwanzig Jahre danach. Deutschland 1945-1965. Deutsche AuBenpolitik, Sonderheft 1, Berlin 1965, S. 106-114.

Semirjaga, Michail: Aspekte sowjetischer Besatzungspolitik in Deutschland nach dem Zweiten Weltkrieg, in: Jacobsen, Hans-Adolf und andere (Hrsg.): Deutsch-russische Zeitenwende. Krieg und Frieden 1941-1995, Baden-Baden 1994, S. 563-587.

Semirjaga, Michail: Kak my upravljali Germaniej [Wie wir Deutschland verwalteten], Moskva 1995.

Semirjaga, Michail: Wie Berijas Leute in Ostdeutschland die „Demokratie“ errichteten, in: DA 29/1996, S. 741-752.

Semjonow, Wladimir S.: Von Stalin bis Gorbatschow. Ein halbes Jahrhundert in diplomatischer Mission, 1939-1991, Berlin 1995.

Sherman, Georg F.: The Russians and the East German Party (prelude to June 17th 1953) London 1956.

Shukow, Georgi K.: Erinnerungen und Gedanken, Bd. I und II, 8. Aufl., Berlin 1987.

Shulman, Marshall D.: Stalin's Foreign Policy Reappraised, New York 1963.

Simonow, Konstantin: Aus der Sicht meiner Generation. Gedanken über Stalin, Berlin 1990.

Slusser, Robert M. (Hrsg.): Soviet Economic Policy in Postwar Germany. A Collection of Papers by Former Soviet Officials, New York 1953. 
SSSR i Germanskij vopros 1941-1949. Die UdSSR und die deutsche Frage 1941-1949. Dokumente aus dem Archiv für Außenpolitik der Russischen Föderation, Bd. 1, 22.6.1941-8.5.1945, Moskva 1996.

Staritz, Dietrich: Die Gründung der DDR. Von der sowjetischen Besatzungsherrschaft zum sozialistischen Staat, München 1984.

Staritz, Dietrich: Die SED, Stalin und die Gründung der DDR. Aus den Akten des Zentralen Parteiarchivs, in: Aus Politik und Zeitgeschichte, Beilage zur Wochenzeitschrift Das Parlament, B 5/1991, S. 3-16.

Staritz, Dietrich: Die SED, Stalin und der „Aufbau des Sozialismus“ in der DDR. Aus den Akten des Zentralen Parteiarchivs, in: DA 24/1991, S. 686-700.

Staritz, Dietrich: Geschichte der DDR 1949-1985, Frankfurt/Main 1985.

Staritz, Dietrich: Zwischen Ostintegration und nationaler Verpflichtung. Zur Ost- und Deutschlandpolitik der SED 1948-1952, in: Herbst, Ludolf (Hrsg.): Westdeutschland 1945-1955. Unterwerfung, Kontrolle, Integration, München 1986, S. 279-289.

Steiniger, Rolf: Eine vertane Chance. Die Stalin-Note vom 10. Mărz 1952 und die Wiedervereinigung, Berlin/Bonn 1985

Stern, Carola: Porträt einer bolschewistischen Partei. Entwicklung, Funktion und Situation der SED, Köln 1957.

Stern, Carola: Ulbricht - eine politische Biographie, Köln 1967.

Stöckigt, Rolf: Direktiven aus Moskau, Sowjetische Einflußnahme auf DDR-Politik 1952/53, in: Briiche, Krisen, Wendepunkte. Neubefragung von DDR-Geschichte, Leipzig und andere, 1990, S. 81-88.

Stöckigt, Rolf: Ein Dokument von historischer Bedeutung vom Mai 1953, in: BzG 32/1990, S. 648-654.

Strunk, Peter: Die Sowjetische Militäradministration in Deutschland (SMAD), in: Lemberg, Hans (Hrsg.), Sowjetisches Modell und nationale Prägung, Marburg 1991, S. 143-176.

Strunk, Peter: Zensur und Zensoren. Medienkontrolle und Propagandapolitik unter sowjetischer Besatzungsherrschaft in Deutschland, Berlin 1996.

Stulz-Herrnstadt, Nadja (Hrsg.): Das Hermstadt-Dokument. Das Politbüro der SED und die Geschichte des 17 Juni 1953, Hamburg 1990.

Suckut, Siegfried (Hrsg.): Blockpolitik in der SBZ/DDR 1945-1949. Die Sitzungsprotokolle des zentralen Einheitsfront-Ausschusses, Köln 1986.

Suckut, Siegfried: Die Entscheidung zur Gründung der DDR. Protokolle der Beratungen des SED-Parteivorstandes am 4. und 9. Oktober 1949, in: VfZ 39/1991, S. 125-175.

Suckut, Siegfried: „Wenn die Nation erhalten bleibt, werden alle administrativen Spaltungsmaßnahmen eines Tages zergehen und zerfallen". Zur Vorgeschichte der DDR-Gründung, in: Die Deutschlandfrage von Jalta und Potsdam bis zur staatlichen Teilung Deutschlands 1949 (Studien zur Deutschlandfrage, Bd. 12), Berlin 1993, S. 117-186.

Suckut, Siegfried: Innenpolitische Aspekte der DDR-Gründung, in: Scherstjanoi, Provisorium, S. 84-101.

Sudoplatow, Pawel Anatoljewitsch/Sudoplatow, Anatolij: Der Handlanger der Macht. Enthüllungen eines KGB-Generals, Diisseldorf u.a. 1994.

SVAG. Upravlenije propagandy (informacii) i S. I. Tjulpanov. 1945 -1949. Sbornik Dokumentov. [Die SMAD. Die Propaganda(Informations-)Verwaltung und S.I. Tjulpanow. 1945-1949. Dokumente], Redakteure Bernd Bonwetsch, Gennadij Bordjugov, Norman Naimark, Moskva 1994.

The Post-Stalin Succession Struggle and the 17 June 1953 Uprising in East Germany: The Hidden History, edited by Christian F. Ostermann for The Cold War International History Project of the Woodrow Wilson International Center for Scholars and the National Security Archive at the George Washington University, An International Conference "The Crisis Year 1953 and the Cold War in Europe", 10.-12. November 1996.

Thoß, Bruno (Hrsg.): Volksarmee schaffen - ohne Geschrei! Studien zu den Anfängen einer „verdeckten Aufrüstung" in der SBZ/DDR 1947-1952, München 1994.

Timofejewa, Natalia: Die deutsche Intelligenz und die geistig-kulturelle Umgestaltung in der SBZ, in: BzG 37/1995, Heft 2, S. 21-33.

Titow, Fjodor Dmitrijewitsch: Sowjetische Juristen unterstützten den Aufbau und die Entwicklung antifaschistisch-demokratischer Justizorgane in der DDR, in: Neue Justiz 39 (1985), Heft 5, S. $185 \mathrm{f}$.

Tjulpanow, Sergej: Deutschland nach dem Kriege (1945-1949). Erinnerungen eines Offiziers der Sowjetarmee, Berlin 1986. 
Tschörtner, llse: „Die Seelen der Deutschen erobern“. Über die Kulturoffiziere der SMAD, in: Berliner Debatte 1994, Heft 1, S. 99-112.

Ulam, Adam B.: Expansion an Co-Existence. The History of Soviet Foreign Policy, 1917-1967, New York, Washington 1968.

Ulbricht, Walter: Zur Geschichte der Deutschen Arbeiterbewegung. Aus Reden und Aufsätzen, Bd. IV: 19501954, Berlin 1958.

Vtoraja mirovaja vojna. Aktual'nye problemy [Der Zweite Weltkrieg. Aktuelle Probleme], Moskva 1995.

Viskov S. I./Kul"bakin V. D.: Sojuzniki i „Germanskij vopros" 1945-1949gg [Die Alliierten und die Deutsche Frage" 1945-1949], Moskva 1990.

Walther, Peter Th.: Fritz Hartung und die Umgestaltung der historischen Forschung an der Deutschen Akademie der Wissenschaften zu Berlin, in: Sabrow, Martin/Walter, Peter Th., Historische Forschung und sozialistische Diktatur. Beiträge zur Geschichtswissenschaft der DDR, Leipzig 1995, S. 59-73.

Wandschneider, Hermann: Zu einigen Aspekten der Entwicklung des Kampfbundes zwischen der SED und der KPdSU und der brüderlichen Verbundenheit der Arbeiterklasse der DDR und der UdSSR in der ersten Hälfte der fünfziger Jahre, in: Die Entwicklung, S. 215-223.

Weber, Hermann (Hrsg.) Parteiensystem zwischen Demokratie und Volksdemokratie. Dokumente und Materialien zum Funktionswandel der Parteien und Massenorganisationen in der SBZ/DDR, Köln 1982.

Weber, Hermann (Hrsg.): DDR. Dokumente zur Geschichte der Deutschen Demokratischen Republik 19451949, München 1986.

Weber, Hermann/Staritz, Dietrich (Hrsg.): Kommunisten verfolgen Kommunisten. Stalinistischer Terror und „Säuberungen" in den kommunistischen Parteien Europas seit den dreißiger Jahren, Berlin 1993.

Wehner, Marcus: Geheime Verschlußsache. Die jüngste Wende der russischen Archivpolitik, in: Frankfurter Allgemeine Zeitung, 12.7.1995.

Wenzke, Rüdiger: Auf dem Wege zur Kaderarmee. Aspekte der Rekrutierung, Sozialstruktur und personellen Entwicklung des entstehenden Militärs in der SBZ/DDR bis 1952/53, in: ThoB, Volksarmee, S. 205-272.

Wettig, Gerhard: Die beginnende Umorientierung der sowjetischen Deutschland-Politik im Frühjahr und Sommer 1953, in: DA 28/1995, S. 495-507.

Wettig, Gerhard: Sowjetische Wiedervereinigungsbemühungen im ausgehenden Frühjahr 1953?, in: DA 25/1992, S. 942-958.

Wettig, Gerhard: Did Beria Seek Agreement on German Unification? An Old Problem in the Light of New Evidence, Vortrag auf der Konferenz „Das Krisenjahr 1953 und der Kalte Krieg in Europa“, Potsdam, 10.-12. November 1996.

Wettig, Gerhard: Zum Stand der Forschung über Berijas Deutschland-Politik im Frühjahr 1953, in: Deutschlandfrage, S. 183-200.

Wettig, Gerhard: Zum Stand der Forschung über Berijas Deutschland-Politik im Frühjahr 1953, in DA 26/1993, S. 674-682.

Zeidler, Stephan: Entstehung und Entwicklung der Ost-CDU 1945-1989. Zum Wandlungs- und Gleichschaltungsprozeß einer Blockpartei, in: Aus Politik und Zeitgeschichte, Beilage zur Wochenzeitschrift Das Parlament, B 16-17/96, S. 22-30.

Zeitzeugenberichte [Wolfgang Schollwer (LDPD), Günther Wirth (CDU), Artur Pommerenke (NDPD)], in: Frölich, Jürgen (Hrsg.): „Bürgerliche“ Parteien in der SBZ/DDR. Zur Geschichte von CDU, LDP(D), DBD und NDPD 1945 bis 1953, Köln 1995, S. 121-143.

Zink, Harold: The United States in Germany, 1944-1953, Princeton 1957.

Zubok, Vladislav M.: „Unacceptably Rude And Blatant On The German Question.“. The Succession Struggle after Stalin's Death, Beria and the Significance of the Debate on the GDR in Moscow in April-May 1953, Vortrag auf der Konferenz „Das Krisenjahr 1953 und der Kalte Krieg in Europa“, Potsdam, 10.-12. November 1996. 
